\title{
Aprovechamiento del recurso maderable tropical nativo en la comunidad de Emilio Rabasa, Reserva de la Biosfera Selva El Ocote, Chiapas, México
}

\author{
Native tropical timber resource use in the community \\ of Emilio Rabasa, at the Selva El Ocote Biosphere Reserve \\ in Chiapas, Mexico
}

\author{
Carolina Orantes-García, ${ }^{1}$ Miguel Ángel Pérez-Farrera, ${ }^{1}$ \\ Carlos Uriel del Carpio-Penagos ${ }^{2}$ y César Tejeda-Cruz ${ }^{3}$
}

\begin{abstract}
RESUMEN
El presente estudio tuvo como finalidad conocer el aprovechamiento de los recursos maderables tropicales nativos por parte de la comunidad Emilio Rabasa, ubicada en la Reserva de la Biosfera Selva El Ocote, en el estado de Chiapas, México. Se realizaron encuestas semiestructuradas a 30 ejidatarios y se colectaron ejemplares para la identificación de las especies. Se determinó un total de 35 especies maderables nativas, incluidas en 31 géneros y 24 familias. Las principales especies maderables multipropósito mencionadas fueron el bojón (Cordia alliodora (R. \& P.) Oken), el palo amarillo (Terminalia obovata (R. \& P.) Steud) la caoba (Swietenia macrophylla G. King), el jolocín (Heliocarpus donnell-smithii Rose), el cedro (Cedrela odorata M. Roem. King) y el copalchi (Croton guatemalensis Lotsy). Entre los principales usos que la comunidad le da a las especies maderables está, 38\% la extracción de leña y postes para cercado, 17\% para la elaboración de herramientas de trabajo y $16 \%$ para la elaboración de muebles, construcción y reparación de casas.
\end{abstract}

PALABRAS CLAVE

Árboles multipropósito, flora nativa, recurso forestal, selva Zoque, trópico de México.

\begin{abstract}
This study aimed to identify the use of native tropical timber resources in the Emilio Rabasa community located in the Ocote Forest Biosphere Reserve, in Chiapas state, Mexico. 30 semi-structured surveys were conducted with ejidatarios "land-owners", and several specimens were collected for their identification. A total of 35 native timber species were determined, included in 31 genera and 24 families. The main multipurpose timber species mentioned were bojón (Cordia alliodora (R. \& P.) Oken), palo amarillo (Terminalia obovata (R. \& P.) Steud), mahogany (Swietenia macrophylla G. King), the jolocin (Heliocarpus donnellsmithii Rose), cedar (Cedrela odorata M. Roem. King) and copalchi (Croton guatemalensis Lotsy). Among the main uses that the community gives these timber species are $38 \%$ for extraction of firewood and fence posts, $17 \%$ for making tools and $16 \%$ for making furniture, construction and houses repair.
\end{abstract}

KEY WORDS

Multipurpose trees, native flora, resources, Zoque forest, tropic of Mexico. 


\section{INTRODUCCIÓN}

México es uno de los países del mundo con mayor diversidad biológica y cultural; se ubica entre los cinco primeros países llamados "megadiversos", los cuales albergan entre $60 \%$ y $70 \%$ de la diversidad biológica conocida del planeta (Mittermeier et al., 2000; Francis y Currie 2003; Conabio, 2006). Posee $10 \%$ de la flora del mundo, con un estimativo de 30000 especies de plantas vasculares, ocupando el cuarto lugar a nivel mundial en riqueza de plantas endémicas y nativas (Villaseñor y Espinosa-García, 2004; Conabio, 2006). De acuerdo con Rzedowski (1992), en el territorio mexicano se encuentran alrededor de 2500 especies de árboles nativos, que constituyen un reservorio de recursos bióticos de valor actual y potencial por los múltiples productos y beneficios ambientales que de ellos se derivan, motivo por el cual su presencia y permanencia es fundamental para contrarrestar los efectos de la deforestación así como para garantizar la sustentabilidad de la tierra (Gligo, 1993; Vázquez et al., 1999; Mardocheo et al., 2001).

Aunque en la actualidad la gestión sostenible de los bosques ha sido ampliamente adoptada como una política y un objetivo de la administración pública, las altas tasas de pérdida y degradación del bosque se siguen produciendo en muchas áreas. Las actividades humanas como la tala, la ganadería, los cultivos, el desarrollo de infraestructura y el uso del fuego, están causando la pérdida generalizada de biodiversidad, restringiendo el progreso del manejo sostenible de los bosques. Sólo en México, la tasa anual de deforestación (237 000 ha) es la causa principal de la pérdida de vegetación en los ecosistemas tropicales (Viana et al., 1997; Witmore, 1997; Dirzo, 2001). Ante tal problemática, existe una urgente necesidad de herramientas que logren proporcionar una evaluación integrada de los impactos humanos sobre la diversidad biológica forestal y que puedan apoyar la toma de decisiones relacionadas con el uso del bosque (Newton et al., 2009).

La Reserva de la Biosfera Selva El Ocote (Rebiso) en el estado de Chiapas (estado cuya riqueza arbórea es de 1400 a 1500 taxa; González-Espinosa et al., 2004), con una superficie total de 101288 - 15 - 12,5 ha (Semarnat, 2000), es considerada de especial importancia biológica y cultural por su cercanía con las selvas de los Chimalapas en Oaxaca y Uxpanapa en Veracruz, que en su conjunto forman un corredor biológico que constituye la selva zoque (Gómez-Pompa y Dirzo, 1995). La Rebiso protege uno de los centros de diversidad biológica más importantes de México y del mundo, ya que se encuentra ubicada en una zona de transición de dos provincias neotropicales, la Pacifiquense y la Tehuantepecana (Vásquez y March, 1996). Además, es un espacio donde se satisfacen necesidades básicas de comunidades campesinas locales, para lo cual hacen uso de los recursos forestales nativos, lo que puede ocasionar un deterioro de dichos recursos (Moreno, 2009). En la Rebiso están presentes numerosas especies forestales de importancia económica (maderable, medicinal, comestible, forrajera y ornamental), no obstante, hoy en día no se cuenta con estudios exhaustivos al respecto. Las contribuciones al conocimiento de dicha flora son todavía incipientes, pocos son los autores (Miranda, 1952; Vásquez-Sánchez, 1988 y Ochoa-Gaona, 1996) que han llevado a cabo algunos trabajos, principalmente estudios florísticos. El presente trabajo es el primer estudio que registra el aprovechamiento del recurso maderable nativo dentro de la Rebiso, como un primer paso para establecer programas y evaluaciones integrales del manejo de estas especies por los habitantes de las comunidades asentadas en el área de amortiguamiento de la misma. 


\section{OBJETIVO}

El presente estudio tuvo como finalidad conocer el aprovechamiento de los recursos maderables tropicales nativos por parte de la comunidad de Emilio Rabasa, localizada en la Reserva de la Biosfera Selva El Ocote, en el estado de Chiapas, México.

\section{MATERIALES Y MÉTODO}

\section{Área de estudio}

La Rebiso se ubica dentro de los municipios de Cintalapa, Ocozocoautla, Tecpatán y Jiquipilas, en el estado de Chiapas, México, entre los paralelos $16^{\circ} 45^{\prime} 42^{\prime \prime}$ y $17^{\circ} 09^{\prime} 00^{\prime \prime}$ de latitud norte y entre los meridianos $93^{\circ} 21^{\prime} 20^{\prime \prime}$ y $93^{\circ} 47^{\prime} 00^{\prime \prime}$ de longitud oeste (Semarnat, 2000). Dentro de la zona de amortiguamiento de la Rebiso se encuentra la localidad de estudio, Emilio Rabasa, la cual pertenece al municipio de Ocozocoautla (Fig. 1). El paisaje predominante en la zona corresponde al de las montañas del norte, constituidas por sierras y serranías de altitud variable entre los $750 \mathrm{msnm}$ y $1500 \mathrm{msnm}$. De acuerdo con la clasificación de Köppen, modificada por García (1980), el grupo climático presente en la zona corresponde al cálido subhúmedo con lluvias abundantes en verano $\left(A w_{1}\right)$ y con una precipitación total anual que varía entre $1500 \mathrm{~mm}$ y 2500 $\mathrm{mm}$, con una temperatura media anual de $22{ }^{\circ} \mathrm{C}$.

Según Rzedowski (1994), la vegetación presente en la Rebiso corresponde a cuatro asociaciones vegetales:

a) Selva alta perennifolia, predominan las especies de chicozapote (Manilkara zapota (L.) Van Royen), caoba (Swietenia macrophylla G. King), cedro (Cedrela odorata M. Roem. King) y mojú (Brosimum alicastrum Sw.). b) Selva alta o mediana subperennifolia, se encuentran especies como el molinillo (Quararibea gentlei Lundell), el copalillo (Bursera bipinnata (Sessé \& Mociño) Engl.), el palo mulato (Bursera simaruba (L.) Sarg.) y el frijolillo (PitheceIlobium arboreum (L.) Urban).

c) Selva mediana o baja perennifolia, las especies representativas son: palo de coleto (Oreopanax peltatus Linden ex Regel) y memelita (Clusia flava Jacq.).

d) Vegetación secundaria, en donde las especies representativas son el corcho colorado (Belotia mexicana (DC.) Schum.), la yerba buena de monte (Pilea pubescens Liebm.), el guarumbo (Cecropia peltata L.) y el macús (Calathea allouia Aubl.).

De acuerdo con la información proporcionada por los habitantes de la comunidad de Emilio Rabasa, los primeros pobladores llegaron en 1970 en busca de terrenos para la agricultura, provenientes de los municipios de Villaflores, Ocozocoautla, Jiquipilas y Tecpatán, Chiapas. En 1981 se fundó el ejido Emilio Rabasa a través de un decreto federal, con una extensión de 840 ha. La comunidad está integrada por 150 habitantes 82 mujeres y 68 hombres. Los habitantes son mestizos, hablan castellano y $70 \%$ saben leer y escribir. Noventa por ciento de los habitantes de la comunidad son ejidatarios que tienen en promedio de 20 ha a 30 ha de terreno cada uno, mientras que el $10 \%$ restante son personas que viven en la comunidad que sólo poseen una casa y un solar.

Dentro de las actividades económicas de la comunidad se encuentra la agricultura, principalmente la siembra de maíz (Zea mays L.) y frijol (Phaseolus vulgaris L.), que es practicada por $37 \%$ de los productores; $32 \%$ se dedica a la producción de café (Coffea arabica L.), 22\% a la apicultura y únicamente $9 \%$ practica la gana- 


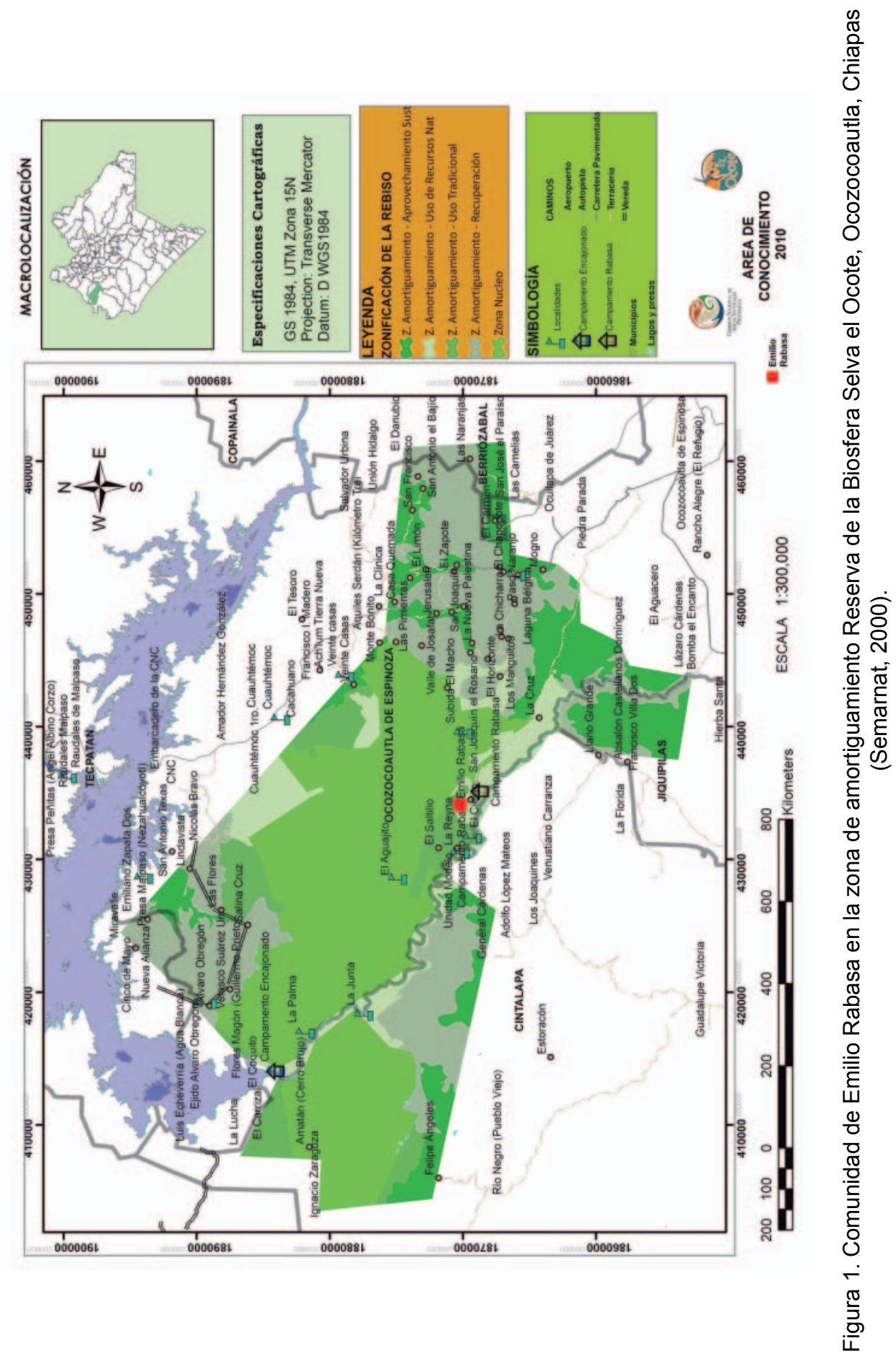


dería ovina; tales actividades son para autoconsumo. La cantidad de terreno destinada a la producción por cada productor oscila entre las 2 ha y 3 ha con un rendimiento de 800 kg/ha para el maíz y 500 $\mathrm{kg} / \mathrm{ha}$ para el frijol. El cultivo del café (Coffea arabica) es la segunda actividad productiva comercial en importancia dentro de la comunidad por la superficie ocupada, el rendimiento promedio de este cultivo es de $700 \mathrm{~kg} / \mathrm{ha}$ a $1000 \mathrm{~kg} / \mathrm{ha}$ de café convencional. El rendimiento ha aumentado con la utilización de técnicas orgánicas, la comercialización la realizan con introductores externos.

La apicultura constituye una alternativa productiva complementaria a la agricultura, al mismo tiempo que es una actividad compatible con la conservación de los recursos forestales de la comunidad. El producto principal es la miel, que es vendida a productores externos de la cabecera municipal de Ocozocoautla, Chiapas. Actualmente los productores están en un proceso de capacitación y ampliación.

\section{Método}

Se aplicaron encuestas semiestructuradas para la recopilación de la información (Geilfus, 1997). Las encuestas se realizaron a los 30 ejidatarios de Emilio Rabasa, con edades entre los 20 y 70 años. Esta herramienta permitió indagar con respecto a las formas de uso del recurso forestal maderable, especies utilizadas, técnicas empleadas y percepción. El trabajo se acompañó de la observación directa así como de conversaciones y cuatro recorridos por las zonas de aprovechamiento junto con los informantes. Los datos recopilados se registraron en una hoja de cálculo de Microsoft Excel para facilitar su análisis, mediante una estadística descriptiva.

De acuerdo con la clasificación de Rzedowski (1994), se determinó el tipo de vegetación donde se encontraron las especies maderables nativas. Siguiendo la metodología de Lot y Chiang (1986), se realizaron colectas de ejemplares de las especies arbóreas nativas para su posterior identificación, con ayuda de bibliografía especializada (Martínez, 1979; Breedlove, 1981; Niembro, 1986; Rzedowski, 1992; Gentry, 1996; Miranda, 1998; Ricker y Douglas, 1998; Pennington y Sarukhán, 2005; Flora Mesoamericana, 2006), los ejemplares identificados fueron depositados en el Herbario Eizi Matuda (HEM) de la Universidad de Ciencias y Artes de Chiapas.

\section{RESULTADOS}

\section{Historia de aprovechamiento}

De acuerdo con las encuestas, el aprovechamiento forestal dio inicio en 1970 cuando los primeros pobladores provenientes de los municipios de Villaflores, Ocozocoautla, Jiquipilas y Tecpatán, Chiapas se establecieron en los terrenos selváticos en los que actualmente se ubica la comunidad. Estos primeros pobladores talaban y quemaban las especies maderables nativas para abrir campos de siembra de cultivos como el maíz ( $Z$. mays.) y frijol ( $P$. vulgaris); aunado a ello, la madera sólo se destinaba para la construcción de las casas y no se comercializaba debido a que no existían caminos para transportarla.

A inicios de 1975, el aprovechamiento forestal maderable se intensificó con la apertura de la carretera y el establecimiento de un aserradero privado dentro de los terrenos de la comunidad. A partir de ello, los habitantes de la comunidad pudieron vender los árboles de maderas preciosas como el cedro (Cedrela odorata) y la caoba (Swietenia macrophylla) a la empresa maderera. En 1980, después de haber cerrado el aserradero, los habitantes 
de la comunidad comenzaron a explotar la madera como principal actividad, misma que presentó una alternativa de generación de recursos económicos inmediatos. Dicha madera era comprada, principalmente por introductores provenientes de la cabecera municipal de Ocozocoautla. Actualmente, la explotación de la madera está regulada por la misma comunidad a través de un reglamento interno y por las instituciones vinculadas con este sector; la madera es utilizada en la mayoría de los casos para reparación de las casas, leña y cercado; existen programas de reforestación en zonas degradadas.

\section{Aprovechamiento forestal}

A través de las encuestas se registró un total de 35 especies maderables nativas, distribuidas en 31 géneros y 24 familias (Tabla 1). Ochenta por ciento $(n=35)$ se encuentran distribuidas en selva alta perennifolia y el $20 \%$ restante en la selva mediana subperennifolia.

Tabla 1. Número total de familias, géneros y especies forestales maderables nativas aprovechadas en el ejido Emilio Rabasa, Rebiso, Chiapas.

\begin{tabular}{lcc}
\hline Familias & Géneros & Especies \\
\hline Amaranthaceae & 1 & 1 \\
Bombacaceae & 2 & 2 \\
Boraginaceae & 1 & 1 \\
Burseraceae & 1 & 3 \\
Cochlospermaceae & 1 & 1 \\
Combretaceae & 1 & 1 \\
Euphorbiaceae & 1 & 1 \\
Fabaceae & 3 & 3 \\
Flacurtiaceae & 1 & 1 \\
Lauraceae & 1 & 1 \\
Meliaceae & 3 & 5 \\
Moraceae & 1 & 1 \\
Myrsinaceae & 1 & 1 \\
Myrtaceae & 1 & 1 \\
Ochnaceae & 1 & 1 \\
Polygonaceae & 1 & 1 \\
Ramnaceae & 1 & 1 \\
Rubiaceae & 2 & 2 \\
Rutaceae & 1 & 1 \\
Sapindaceae & 1 & 1 \\
Sapotaceae & 1 & 1 \\
Simaroubaceae & 1 & 1 \\
Tiliaceae & 1 & 1 \\
Ulmaceae & 2 & 2 \\
\hline TOTAL 24 & 31 & 35 \\
\hline
\end{tabular}


De acuerdo con los encuestados, en Emilio Rabasa, 70\% ( $n=35)$ de las especies maderables utilizadas son multipropósito (Tabla 2). Se registraron 10 usos que se les da a las especies: leña, postes, cercas vivas, construcción de casas, elaboración de muebles, herramientas de trabajo, medicinal, comestibles, melíferas y forrajeras, en donde $38 \%(n=35)$ de las especies son utilizadas en la extracción de leña y postes para cercado, $17 \%$ para la elaboración de herramientas de trabajo, $16 \%$ para la elaboración de muebles, construcción y reparación de casas, $12 \%$ son melíferas, $8 \%$ son utilizadas en la medicina tradicional, $5 \%$ como cercos vivos y $4 \%$ como comestible y forrajera.

De 43\% ( $n=35)$ de la especies maderables nativas se utilizan sólo las ramas y troncos para la obtención de leña, cercado de corrales y herramientas de trabajo, de $16 \%$ se usa la hoja, tal es el caso de la colita de borrego (Picramnia sp.),la pimienta (Pimienta dioica), cuyas hojas son utilizadas en la medicina tradicional y el mojú (Brosimum alicastrum) que se aprovecha como forraje; de $15 \%$ de los árboles como el bojón (Cordia alliodora), el palo amarillo (Terminalia obovata), la caoba (Swietenia macrophylla), el cedro (Cedrela odorata) y el hormiguillo (Platymiscium dimorphandrum) se utiliza todo el árbol para diferentes fines; de $11 \%$ se aprovecha la corteza, como el palo mulato (Bursera simaruba), el copalillo (Bursera bipinnata) y el copal (Bursera excelsa); de $8 \%$ de las especies se utiliza únicamente la flor, como el sospó (Pseudobombax ellipticum) y el pumposhuiti (Cochlospermum vitifolium); y de $7 \%$ de los árboles maderables nativos se aprovechan los frutos y las semillas, tal es el caso del mojú (Brosimum alicastrum), el chicozapote (Manilkara zapota) y la pimienta (Pimienta dioica).

Las especies como el cedro (Cedrela odorata), la caoba (Swietenia macro- phylla), el bojón (Cordia alliodora), el mojú (Brosimum alicastrum), el palo amarillo (Terminalia obovata), el jolocín (Heliocarpus donnell-smithii) y el copalchi (Croton guatemalensis), son las que presentan mayor frecuencia de uso $(100 \%, n=30)$, mientras que especies como el espino (Acacia pennatula L.), el frijolillo (Pithecellobium arboreum (L.) Urban), el pumposhuiti (Cochlospermum vitifolium Willd. ex Spreng) y el sospó (Pseudobombax ellipticum (H.B.K.) Dugand) son utilizadas en menor medida (10\%) por los habitantes de la comunidad.

De acuerdo con los encuestados, en el ejido Emilio Rabasa, los hombres integrantes del núcleo familiar realizan el aprovechamiento de las especies maderables nativas. La madera es cortada in situ; para la extracción de leña y postes, la madera es cortada mediante hacha y llevada a la comunidad por las mujeres, mientras que para la construcción de viviendas, fabricación de muebles y puertas, se utiliza principalmente la motosierra y es transportada mediante arrastre por caballos.

Los árboles son seleccionados según el uso; para leña, postes y herramientas de trabajo se seleccionan árboles de 2 años o más, con d.a.p. (diámetro a la altura del pecho) entre $0,10 \mathrm{~m}$ y $0,30 \mathrm{~m}$, altura de $2 \mathrm{~m}$ a $5 \mathrm{~m}$; para construcción de casas y muebles se seleccionan árboles de 5 años en adelante, con d.a.p. de 0,50 $\mathrm{m}$ a $1,50 \mathrm{~m}$ y altura de $15 \mathrm{~m}$ a $40 \mathrm{~m}$.

Los encuestados también indicaron que en la comunidad la venta de madera es casi nula, ya que dicha actividad está regulada por la misma comunidad a través de un reglamento interno para la explotación forestal y por las instituciones vinculadas con este sector (Profepa, Conanp y Semarnap). Sin embargo, también aclararon que aún existen pobladores que venden árboles en pie, con precios que van de MXP\$1000 a MXP\$4000, con 


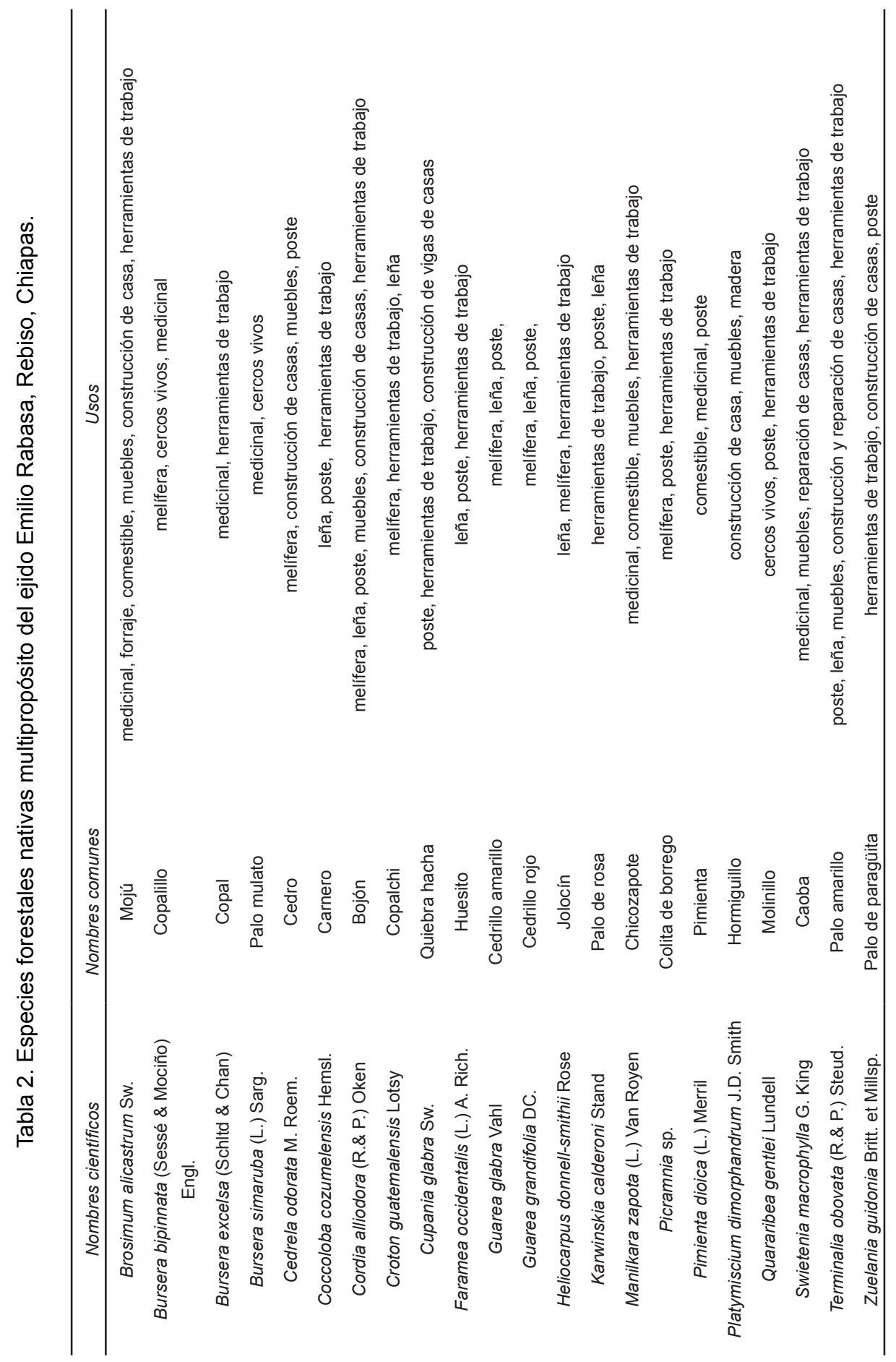


compradores de la cabecera municipal de Ocozo-coautla, Chiapas. Las principales especies arbóreas nativas que se comercializan se enlistan en la tabla 3.

\section{DISCUSIÓN}

En Emilio Rabasa, más de $70 \%$ de los árboles nativos se aprovechan para la extracción de leña, elaboración de herramientas de trabajo, postes para cercado, elaboración de muebles, construcción y reparación de casas. Estos resultados coinciden con Torres (2004), al sugerir que el uso de leña para el hogar representa, en pleno siglo XXI, $40 \%$ de la demanda de energía doméstica nacional.

Las especies arbóreas nativas encontradas en Emilio Rabasa como el cedro (Cedrela odorata), la caoba (Swietenia macrophylla), el bojón (Cordia alliodora), el chicozapote (Manilkara zapota), el mojú (Brosimum alicastrum) y el palo amarillo (Terminalia obovata), son mencionadas en diversos estudios como especies multipropósito para reforestación, restauración y plantaciones forestales. Entre estos estudios se pueden señalar el realizado en el área de Jurisdicción, CDMB (Corporación Autónoma Regional para la Defensa de la Meseta de Bucaramanga), Santander, Colombia, (2002) en el que se llevó a cabo una evaluación para determinar el potencial de especies forestales nativas para la reforestación, identificándose algunas especies forestales nativas promisorias en diferentes formaciones ecológicas. Entre estas especies se encuentran el frijolito (Schizolobium parahybum), cedro (Cedrela odorata), anime (Protium sp.), pardillo (Cordia alliodora), balso (Ochroma pyramidale), caimo (Pouteria sp.), cedrón (Simaba cedron), chingalé (Jacaranda copaia) y ojite (Brosimun alicastrum), entre otras.

Tabla 3. Especies arbóreas nativas comercializadas, mencionadas por los habitantes de Emilio Rabasa.

\begin{tabular}{ccc}
\hline Nombre científico & Nombres comunes & Uso \\
Cedrela odorata & Cedro & Muebles, puertas \\
Coccoloba cozumelensis & Carnero & Leña, poste \\
Cordia alliodora & Bojón & Muebles, construcción \\
Croton guatemalensis & Copalchi & Poste \\
Faramea occidentalis & Huesito & Leña \\
Heliocarpus donnell-smithii & Jolocín & Leña \\
Swietenia macrophylla & Caoba & Muebles, puertas \\
Terminalia obovata & Palo amarillo & Muebles, construcción \\
Zuelania guidonia & Palo de paragüita & Poste \\
\hline
\end{tabular}


Por su parte, Rivas (2003) explicó que en El Salvador se realizaron importantes investigaciones sobre el establecimiento y manejo de plantaciones forestales en las que se identificaron 49 especies forestales con potencial productivo; entre las especies que se mencionan en dichos trabajos están el almendro de río (Andira inermis), balsa (Ochroma lagopus), bálsamo (Miroxylon balsamun), barillo (Calophylum brasiliense), caoba (Swietenia macrophylla) carao (Cassia grandis), caulote (Guazuma ulmifolia), cedro (Cedrela odorata) y pajarito (Cordia alliodora). De ellas, S. macrophylla, C. Odorata y C. alliodora, fueron especies con mayor número de menciones en la comunidad Emilio Rabasa.

Por su parte, el CATIE (2006) llevó a cabo el proyecto llamado "Developing best practices for seed sourcing of planted and natural regeneration in theneotropics", conocido como Proyecto Seedsource, en la región comprendida desde el sur de México hasta Panamá. En dicho proyecto se registraron un total de 50 especies forestales prioritarias del trópico americano, de las cuales la caoba (Swietenia macrophylla), el cedro (Cedrela odorata), el pajarito (Cordia alliodora) y el copalchi (Croton guatemalensis) resultaron tener la mayor demanda para fines de reforestación comercial. Estas especies coinciden con las mencionadas por los habitantes de la comunidad aquí estudiada como especies de maderables nativas que son comercializadas a escala local.

Otros trabajos que concuerdan con las especies multipropósito encontradas en la comunidad en estudio, son los realizados por Wightman et al. (2006) en la Amazonía Peruana, en donde la producción maderera es una de las actividades económicas más importantes. Por ello desarrollaron un manual en el que listan 42 especies vegetales de importancia maderable, de las cuales se estudiaron varios aspectos de cultivo. Entre las especies mencionadas están: cedro (Cedrela odorata), amasisa (Erythrina ulei), bolaina negra (Guazuma ulmifolia), cacao (Theobroma cacao), caoba (Swietenia macrophylla), copalchi (Croton guatemalensis), copaiba (Copaifera officinalis), cumala (Virola calophylla) y estoraque (Myroxylon balsamum).

Igualmente, Palacios et al. (2008) Ilevaron a cabo un estudio sobre el conocimiento del aprovechamiento de los recursos naturales, en las comunidades de Chintadó, Doña Josefa y Yuto, en la cuenca media del río Atrato, Chocó, en Colombia. En dicho estudio encontraron que las principales especies maderables extraídas son caoba (Swietenia macrophylla), chanú (Humiriastrus procenum), cedro (Cedrela odorata) y lirio (Couma macrocarpa). Lo que concuerda con el estudio en la comunidad Emilio Rabasa en donde se encontró a S. macrophylla y Cedrela odorata como especies maderables nativas extraídas.

En México, Rzedowski (1992) encontró la presencia de aproximadamente 9000 especies de árboles y arbustos nativos en el país. De éstos, Vázquez y Cervantes (1993) realizaron una búsqueda bibliográfica de aquellos potencialmente valiosos para la restauración ecológica y la reforestación; durante dicha investigación seleccionaron 233 especies leñosas nativas de México. El criterio para la selección de estas especies se centró básicamente en que fueran especies multipropósito y con características para ser empleadas en programas de restauración y reforestación en las diferentes regiones ecológicas del país. Entre las 233 especies se encuentran Brosimum alicastrum, Bursera bipinnata, Bursera simaruba, Cedrela odorata, Coccoloba cozumelensis, Cordia alliodora, Croton guatemalensis, Faramea occidentales, Swietenia macrophylla, Terminalia obovata, Manil- 
kara zapota, Pimienta dioica, Platymiscium dimorphandrum, mencionadas por los habitantes de Emilio Rabasa como especies multipropósito.

Posteriormente, Benítez et al. (2004) realizaron estudios sobre árboles multiusos nativos de Veracruz para reforestación, restauración y plantaciones, algunas de las especies encontradas fueron el canshán (Terminalia obovata), molinillo (Quararibea gentlei), chicozapote (ManiIkara zapota), caoba (Swietenia macrophylla), guapaque (Dialium guianense), mojú (Brosimum alicastrum), cedro rojo (Cedrela odorata), ceiba (Ceiba pentandra), baqueta (Ulmus mexicana) y sonzapote (Licania platypus). Las cuales también son reportadas en la comunidad de Emilio Rabasa.

Ahora bien, en la comunidad Emilio Rabasa las ramas y los troncos son las partes más usadas de los árboles. El principal aprovechamiento de los árboles se realiza con el fin de obtener materiales para combustible de uso doméstico, construcción y postes para cercados. Ello significa que el uso de la madera sigue siendo fundamental para la vida diaria de las comunidades rurales, proporcionando techo, alimento y apoyando las actividades productivas, y explica el por qué las ramas y troncos son las partes más usadas. Como lo mencionan Palacios et al. (2008), de los árboles nativos se utilizan distintas partes vegetativas, desde semillas, flores, hojas, hasta ramas en porcentajes que van de $10 \%$ a $40 \%$. Sin embargo, la extracción de madera en donde se derriba todo el árbol se realiza en casi $50 \%$ en especies nativas, lo que ha traído como consecuencia el deterioro del recurso forestal.

El potencial de producción de bienes forestales es considerable, se utilizan cientos de productos (hojas, frutos, semillas, cortezas, gomas, ceras, fibras, colo- rantes, entre otros), obtenidos de entre 5000 a 7000 especies distribuidas en los diferentes ecosistemas del territorio nacional (Torres, 2004). La falta de datos estadísticos no permite saber el número exacto de productos que se utilizan ni el valor de la producción. Sin embargo, 20\% de la población más marginada del país, que coincidentemente habita en las zonas rurales, depende para su subsistencia de los productos forestales obtenidos de los ecosistemas en los que viven (Torres, 2004).

En síntesis, existe un conjunto de especies maderables nativas ampliamente mencionadas tanto por la diversidad de usos que tienen, como porque son muy demandadas y porque se usan varias partes de la planta. A partir de estos datos, se sugiere que las especies de Swietenia macrophylla, Cedrela odorata, Brosimum alicastrum, Cordia alliodora, Manilkara zapota,Terminalia obovata, Heliocarpus donnell-smithii, Croton guatemalensis, Bursera simaruba, Coccoloba cozumelensis y Cupania glabra deben ser consideradas para ser utilizadas en programas de restauración, reforestación y plantación forestal. Dos especies que no fueron mencionadas por los habitantes de la comunidad de Emilio Rabasa son Acacia pennatula y Pithecellobium arboreum, sin embargo, éstas han sido utilizadas en otras regiones como especies forrajeras y cercos vivos. Incluso, hoy en día, a nivel mundial se realizan estudios sobre la contribución de $A$. pennatula en la productividad agroforestal sostenible (Nieto et al., 2001), por lo que podrían aprovecharse de igual forma dentro de la Rebiso.

Newton et al. (2009) menciona que la falta de información sobre los procesos ecológicos y características de las especies dificulta el manejo sostenible de los bosques. Por ello, es recomendable que para realizar un aprovechamiento adecuado de las especies forestales aquí cita- 
das, deben realizarse antes estudios sobre germinación, crecimiento y el establecimiento de las especies, así como la evaluación del impacto del aprovechamiento, ya que los cambios en los esquemas de uso de la tierra y los usos y costumbres ligados a ello, pueden significar la pérdida de especies y de la diversidad genética, especialmente de la biota tropical que fueron seleccionadas en función de los ambientes y microhábitats y que hoy perduran en los ecosistemas tropicales (Nasi y Frost, 2009).

\section{CONCLUSIONES}

Las especies maderables nativas aprovechadas en la comunidad de Emilio Rabasa representan $6 \%$ de las especies arbóreas presentes en el área, $23 \%$ del total de las familias de Angiospermas encontradas para la Reserva de la Biosfera Selva El Ocote por Ochoa-Gaona (1996). Dichas especies representan una opción para ser utilizadas en forma integral de una manera económica y ecológicamente viable debido a que se presentan especies multipropósito que pueden ser manejadas en programas de reforestación, restauración y plantaciones, tal como lo sugieren Benítez et al. (2004).

De la lista de 35 especies encontradas en la comunidad de Emilio Rabasa, seis coinciden con las especies de mayor demanda a nivel nacional e internacional: Cedrela odorata, Swietenia macrophylla, Cordia alliodora, Manilkara zapota, Brosimum alicastrum y Terminalia obovata (Rivas, 2003; CATIE, 2006; Wightman et al., 2006).

El presente trabajo muestra que el aprovechamiento del recurso forestal maderable nativo por parte de la comunidad sigue siendo vigente para necesidades básicas de la vida diaria, y que hay un conjunto de especies preferidas. El uso de leña y de postes son dos usos vigentes y, en amplios sectores, también la construcción, de tal modo que se requieren propuestas de reforestación que garanticen el abasto de madera. El uso de postes, impulsando las cercas vivas, puede traer asimismo beneficios ambientales. Sin embargo, es importante la realización de un estudio detallado sobre el impacto ambiental generado por la extracción forestal.

Las selvas tropicales, como la Selva El Ocote, representan un ecosistema único con gran biodiversidad y hábitat exclusivo de muchas plantas y animales en peligro de extinción y son considerados como principales fuentes de madera susceptibles de ser explotados por las comunidades locales (Smouts, 2003; Mittermeier et al., 2003).

\section{RECONOCIMIENTOS}

A los habitantes de la comunidad de Emilio Rabasa por todo el apoyo proporcionado para llevar a cabo la investigación. A COCYTECH-UNICACH por la beca otorgada en la realización del Doctorado en Ciencias de Desarrollo Sustentable, a Fundación Produce Chiapas, A. C., por el financiamiento del proyecto "Identificar y establecer rodales con los mejores árboles semilleros". A la doctora Tamara M. Rioja Paradela, doctor Rubén A. Moreno Moreno y doctor Eduardo R. Garrido Ramírez por la revisión y sugerencias al manuscrito.

\section{REFERENCIAS}

Benítez, G., M.T.P. Pulido-Salas y M. Equihua. 2004. Árboles multiusos nativos de Veracruz para reforestación, restauración y plantaciones. Instituto de Ecología, A.C., Sigolfo, Conafor, Xalapa, Veracruz, México. 288 p. 
Breedlove, D.E. 1981. Flora of Chiapas, Part I: Introduction to the flora of Chiapas. The California Academy of Sciences, San Francisco, California, EUA. 35 p.

CATIE. 2006. Prácticas de recolección, manejo y uso de germoplasma de especies forestales nativas en América Central y sur de México. Proyecto SEEDSOURCE. Informe Final, Cartago, Costa Rica. 62 p.

CDMB. 2002. Programa Reforestar. Bucaramanga, CDMB (Corporación Autónoma

Regional para la Defensa de la Meseta de Bucaramanga), Subdirección de Planeación y Sistemas. 96 p.

Conabio. 2006. Capital natural y bienestar social. $1^{\text {a }}$ ed. Redacta, S.A de C.V., México. $71 \mathrm{p}$.

Dirzo, R. 2001. Tropical forests. In: F. S. Chapin III, O. E. Sala y E. Huber-Sannwald, eds. Global biodiversity in a changing environment. Scenarios for the 21st century. Ecological Studies 152. Springer. Nueva York. p: 251-276.

Francis, A.P. y D.J. Currie. 2003. A globally consistent richness climate relationship for angiosperms. The American Naturalist 161:523-536.

Flora mesoamericana. 2006. http://www. mobot.org/mobot/fm/

García, E. 1980. Modificación del sistema de clasificación climático de Köppen. Instituto de Geografía. UNAM. México. $246 \mathrm{p}$.

Geilfus, F. 1997. 80 herramientas para el desarrollo participativo: diagnóstico, planificación, monitoreo, evaluación. IICA-GTZ. San Salvador, El Salvador. $208 \mathrm{p}$.
Gentry, A. 1996. A field guide to the families and genera of woody plants of Northwest South America. Conservation International. University of Chicago Press, Chicago y Londres. 489 p.

Gligo, N. 1993. Medio ambiente y recursos naturales en el desarrollo Latinoamericano. CEPAL - PNUMA. Editorial Latinoamericana. $72 \mathrm{p}$.

Gómez-Pompa, A. y R. Dirzo. 1995. Reservas de la biosfera y otras áreas naturales protegidas de México. Instituto Nacional de Ecología, Semarnap y Comisión Nacional para el Conocimiento y Uso de la Biodiversidad. 160 p.

González-Espinosa, M., J.M. Rey-Benayas, N. Ramírez-Marcial, M.A. Huston y D. Golicher. 2004. Tree diversity in the northern neotropics: regional patterns in highly diverse Chiapas, México. Ecography 27(6):741-756.

Lot, A y F. Chiang. 1986. Manual de herbario. Administración y manejo de colecciones, técnicas de recolección y preparación de ejemplares de herbario. Consejo Nacional de la Flora de México, A.C. México, D.F. 142 p.

Mardocheo, P., J. Romero y A. Velásquez. 2001. La cuenca de México: una revisión de su importancia biológica. Biodiversitas 37:12-15.

Martínez, M. 1979. Catálogo de nombres vulgares y científicos de plantas mexicanas. Fondo de Cultura Económica. México, D.F. 349 p.

Miranda, F. 1998. La Vegetación de Chiapas. Coneculta. $3^{a}$ ed. Talleres Gráficos del Estado de Chiapas. 205 p.

Miranda, F. 1952. La Selva de El Ocote. Publicaciones del Ateneo de Chiapas. Tuxtla Gutiérrez, Chiapas. 56 p. 
Mittermeier, R.A., N. Myers, J.B. Thomsen, G.A.B. Da Fonseca y S. Oliveiri. 2000. Biodiversity hotspots and major tropical wilderness areas: Approaches to setting conservation priorities. Conservation Biology 12:516-520.

Mittermeier, R.A., C.G. Mittermeier, T.M. Brooks, J.D. Pilgrim, W.R. Konstant, G.A.B. da Fonseca and C. Kormos. 2003. Wilderness and biodiversity conservation. Proceedings of the National Academy of sciences of the United States of America, PNAS 100(18):10309-10313.

Moreno, M.R.A. 2009. Comunidad campesina y apropiación social de los recursos naturales en la selva El Ocote. Tesis de doctorado, Instituto de Ciencias Sociales y Humanidades, Benemérita Universidad Autónoma de Puebla, México. 207 p.

Nasi, R. y P.G.H. Frost. 2009. Sustainable forest management in the tropics: is everything in order but the patient still dying? Ecology and Society 14(2):40. URL: http://www.ecologyandsociety. org/vol14/iss2/art40/

Newton, A.C., L. Cayuela, C. Echeverría, J.J. Armesto, R.F. Del Castillo, D. Golicher, D. Geneletti, M. GonzálezEspinosa, A. Huth, F. López-Barrera, L. Malizia, R. Manson, A. Premoli, N. Ramírez-Marcial, J. Rey Benayas, N. Rüger, C. Smith-Ramírez, y G. Williams-Linera. 2009. Toward integrated analysis of human impacts on forest biodiversity: lessons from Latin America. Ecology and Society 14(2):2. URL: http://www.ecologyandsociety. org/vol14/iss2/art2/

Niembro, R.A. 1986. Árboles y arbustos útiles de México, naturales e introducidos. Limusa. Universidad Autónoma Chapingo. México. 43 p.
Nieto, H., E. Somarriba y M. Gómez. 2001. Contribución de Acacia pennatula (carbón) a la productividad agroforestal sostenible de la Reserva Natural Miraflor-Moropotente, Estelí, Nicaragua. Revista Agroforestería en las Américas 8(30):21-23.

Smouts, M.C. 2003. Tropical forests, international jungle. 1a ed. Palgrave Macmillan, EUA. 244 p.

Ochoa-Gaona, S. 1996. La vegetación de la Reserva El Ocote a lo largo del cañon del río La Venta. In: S.M.A Vásquez, e I. March M. eds. Conservación y desarrollo sustentable en la Selva El Ocote. $1^{\text {a }}$ ed. Conabio, Ecosfera, México. p: 45-86.

Palacios, M.Y., A.B. Rodríguez y O.A.M. Jiménez. 2008. Aprovechamiento de los recursos naturales por parte de la comunidad local en la cuenca media del río Atrato Chocó, Colombia. Revista Institucional Universidad Tecnológica del Chocó; Investigación, Biodiversidad y Desarrollo 27(2):175-185.

Pennington, T.D. y J. Sarukhán. 2005. Árboles tropicales de México. Manual para la identificación de las principales especies. $3^{a}$ ed. Fondo de Cultura Económica, UNAM. México. 522 p.

Ricker, M. y D.C. Douglas. 1998. Botánica económica en bosques tropicales. Principios y métodos para su estudio y aprovechamiento. $1^{\mathrm{a}}$ ed. Diana, México. 293 p.

Rivas, F. 2003. Especies forestales con potencial productivo en El Salvador. Ministerio de Agricultura y Ganadería, Oficina de Políticas y Estrategias Unidad Ambiental Sectorial. 52 p.

Rzedowski, J. 1992. El endemismo en la flora fanerogámica mexicana: una 
apreciación analítica preliminar. In: G. Halffter, eds. Diversidad biológica de Iberoamérica. Acta Zoológica Mexicana. $1^{a}$ ed. Instituto de Ecología, A. C. Xalapa, Veracruz. México. p: 337359.

Rzedowski, J. 1994. Vegetación de México. $6^{a}$ ed. Limusa, México D.F. 432 p.

Semarnat. 2000. Programa de manejo de la Reserva de la Biosfera Selva El Ocote. México, INE-Semarnat. $1^{\text {a }}$ ed. Impresos. México. 220 p.

Torres, R.J.M. 2004. Estudio de tendencias y perspectivas del sector forestal en América Latina al año 2020. FAO, Roma. 145 p.

Vásquez, S.M.A e I. March M. 1996. Conservación y desarrollo sustentable en la Selva El Ocote. $1^{\text {a }}$ ed. Conabio, Ecosfera. México. 475 p.

Vásquez-Sánchez, M. A. 1988. La Selva El Ocote. Monografía y plan de manejo para su conservación. Inireb. Xalapa, México. $70 \mathrm{p}$.

Vázquez, Y.C. y V. Cervantes. 1993. Estrategias para domesticación y propagación de árboles nativos de México. Ciencia y Desarrollo 19(113):52-58.

Vázquez, Y.C., M.A.I Bátis, S.M.I Alcócer, D.M. Gual y D.C. Sánchez. 1999. Árboles y arbustos nativos potencialmente valiosos para la restauración ecológica y la reforestación. Instituto de Ecología. UnAm. Conabio. México, D.F. 311 p.

Viana, V.M., A.J. Tabanez y L.F. Batista, J. 1997. Dynamics and restoration of forest fragments in the Brazilian Atlantic Moist Forest. In: W.F. Laurance y R.O. Bierregaard, eds. Tropical forest remnats. Ecology, management and conservation of fragmented communities. University of Chicago Press. Chicago. p: 351-365.

Villaseñor, J.L. y F.J. Espinosa-García. 2004. The alien flowering plant of México. Diversity and distributions 10(2):113-123.

Wightman, K.E, P. Cornelius J. y L.J. Ugarte. 2006. ¡Plantemos Madera! Manual sobre el establecimiento, manejo y aprovechamiento de plantaciones maderables para productores de la Amazonía peruana. World Agroforestry Centre (ICRAF) Technical Manual no. 4 World Agroforestry Centre - Amazon Regional Programme CIPICRAF, Lima, Perú. 195 p.

Witmore, T.C. 1997. Tropical forest disturbance, disappearance, and species loss. In: W.F. Laurance y R.O. Bierregaard, eds. Tropical forest remnats. Ecology, management and conservation of fragmented communities. University of Chicago Press. Chicago. p: 3-12. recurso maderable tropical nativo en la comunidad de Emilio Rabasa, Reserva de la Biosfera Selva el Ocote, Chiapas, México. Madera y Bosques 19(3):7-21. 